\title{
Equitable Care for Indigenous People: every health service can do it
}

\author{
J Dwyer, K O’Donnell, E Willis and J Kelly
}

\begin{abstract}
Problem and its context: Indigenous peoples in many countries suffer poorer health and poorer access to good healthcare than their non-Indigenous counterparts. In Australia, enduring barriers to good health and good healthcare remain, in spite of long-standing policy priorities. These barriers include the ongoing reality of colonisation, and silence about its implications. People working in and using the health system need to relate across cultures, but they approach this endeavour with a complex mixture of goodwill, defensiveness, guilt and anxiety.
\end{abstract}

Methods: We analysed what is known in Australia about differentials in access to good care, and the underlying factors that entrench them, as well as strategies for developing mainstream competence in care for Aboriginal and Torres Strait Islander patients and communities.

Analysis and Conclusions: The available evidence of differentials in access and quality that are not explained by clinical or demographic variables is unequivocal.

\section{Professor Judith Dwyer}

Flinders University School of Medicine

Dr Kim O'Donnell

Flinders University School of Medicine

Professor Eileen Willis

Flinders University School of Health Sciences

\section{Dr Janet Kelly}

Adelaide University School of Nursing

Correspondence:

Judith.dwyer@flinders.edu.au
Official policy needs to be implemented at the system and organisation level through operational policies, programs and protocols, and through relationships with Aboriginal healthcare providers and community organisations. The concept of racism anxiety provides a way of making one important barrier visible, and moving beyond it can enable people of goodwill to 'see' where change is needed, and to see themselves as part of the solution. It is time to get beyond the barriers and attend to practical improvements in care, focused on the care system, not simply on the skills and knowledge of individuals within it.

Abbreviations: ACCHO - Aboriginal Community Controlled Health Organisation; CC - Cultural Competence.

Key words: Indigenous health; health equity, hospitals; cultural safety; systemic racism.

\section{Introduction}

Indigenous peoples in many countries suffer poorer health and poorer access to good healthcare than their nonIndigenous counterparts. In Australia, enduring barriers to good health and good healthcare remain, in spite of longstanding policy priorities. These barriers include the ongoing reality of colonisation and silence about its implications. People working in and using the health system need to relate across cultures, but they approach this endeavour with a complex mixture of goodwill, defensiveness, guilt and anxiety.

\section{Methods}

We conducted a literature review of Australian evidence on differentials in access to good healthcare affecting Aboriginal and Torres Strait Islander people (henceforth, 
Aboriginal), and on strategies for working towards equitable care. We analysed this evidence in the light of our own research on the challenges facing mainstream healthcare staff in attempting to implement better care.

\section{Differentials in access and quality}

Australian research on differentials in care has established that systemic racism is real, with damaging effects on access and quality. While there is a need for more evidence in particular areas (to inform the health system about opportunities for improvement), the evidence from existing research focused on both various clinical specialties (we address three below) and on indicators of good processes of care is consistent.

\section{Care for cancer}

While the higher cancer mortality of Aboriginal patients is well known (e.g. 2.5 times more likely to die within five years of diagnosis in the Northern Territory) [1] the possible factors underlying this differential are many and complex. A literature review of evidence in relation to barriers to optimal lung cancer care for Aboriginal people [2] identified a combination of individual beliefs and behaviours, healthcare system issues (including discrimination and racism) and the impact of social determinants. These conclusions are supported by Boffa, [3] who identified many practical barriers to access, based partly on assumptions about patients' treatment preference and likely compliance.

\section{Cardiovascular care}

The Heart Foundation and the Australian Healthcare and Hospitals Association [4] identified an inpatient death rate of twice the national average, and a $40 \%$ lower rate of intervention for Aboriginal patients. There is some evidence of improvement in a recent national report on cardiac health, [5] although Aboriginal people still have higher death rates and lower rates of access to effective treatment, with strong regional variations. A qualitative study of Aboriginal cardiac patient journeys [6] identified barriers to use of health services at both organisational and individual levels, including perceptions of interpersonal and institutional racism among patients, families and health care staff.

\section{Kidney care}

Differential access to kidney transplantation [7] is particularly important given the high incidence of kidney disease (eight times the national average, [8]) and the heavy burden of dialysis for patients. As part of a large qualitative study, [9] Anderson et al [10] addressed the views of renal physicians, who reported that they commonly identify Aboriginal patients as both non-compliant and high-risk candidates for kidney transplant. Although the definition and assessment of noncompliance were neither systematic nor based on evidence about the value of compliance in predicting transplant outcomes, some physicians gave considerable weight to compliance and risk in their decision-making. The authors concluded that it is likely that reliance on assessment of compliance by some renal physicians will continue to disadvantage Aboriginal patients with kidney disease.

\section{Other indicators}

Other indicators of differential access to care include longer waiting times for Aboriginal patients to be seen in hospital emergency departments, [11] and for surgery. $[11,12]$ Longer waiting times are one factor that influences Aboriginal people to leave hospital without being treated, or against medical advice.

National data indicates that Aboriginal people were six times more likely to leave hospital without medical discharge; [13] and a regional study in New South Wales describes higher rates of Aboriginal people leaving without treatment, or against medical advice, from rural hospital emergency departments. [14] Self-discharge from inpatient care is also higher for Aboriginal patients, [15] with communication failures prominent among the identified factors influencing this outcome. Most of the Aboriginal patients did not know the reason for their admission or their predicted length of stay. The involvement of Aboriginal Liaison Officers was associated with reduction in self-discharge. The authors conclude that improving cultural safety may be the key.

Disparities have been documented in relation to screening, prevention of complications and potentially preventable hospitalisations, [16] rates of intervention, [17] continuity of care [18] and supportive services such as cardiac rehabilitation. [19] The impacts of past and present experiences of exclusion, shaming and stereotyping; [4,20-23] and language and interpersonal communication difficulties [20,24,25] have also been demonstrated. Experiences of racism in healthcare have been associated with high psychological distress, and have more impact than experiences of racism in other settings. [26]

While evidence of differentials in access and quality of care has been established, and inferences can be drawn about the impact on health outcomes, there are other important causes of poorer health outcomes for Aboriginal people that lie outside the health system (principally, exposure to the negative impact of social and cultural determinants of health). In an influential study of comparative burden of disease, Vos et al [27] showed that disparities are spread 
across all major disease groups, with cardiovascular diseases, injuries, diabetes, mental illness (including substance use disorders) and respiratory diseases contributing the highest excess burdens of illness. While acknowledging the complex causation of these differences, the authors suggest that the higher case fatality rates for most diseases are related to poorer access and poorer quality of care (including late presentation, problems in acute management and poor follow-up). These health policy/care factors also contribute to higher burden of illness for those who survive.

\section{Acknowledging systemic racism}

Evidence of differentials in access to and quality of healthcare that are not explained by clinical or other relevant factors (including disease prevalence and geography) constitutes evidence of systemic or institutional racism. That is, systemic racism is encoded in the policies and funding regimes, healthcare practices and prejudices that affect Aboriginal people's access to good care differentially. It is the impact on health and care, not the intention of policy-makers or care providers, which matters.

With colleagues, we have investigated the gap between high level policies (which seek to re-dress discrimination and disadvantage) and the implementation of effective strategies to enact those policies in practice. [28] While in many ways the question of interest is what to do about it, it is unlikely that such efforts will be well founded without an explicit analysis and understanding of systemic racism and how it works.

It is one thing to recognise and understand the purpose, methods and impacts of systemic racism, and quite another to explicitly and directly confront it in efforts to reduce its impacts on health and mainstream healthcare in practice. Our research with clinical teams who provide care for rural and remote Aboriginal patients found that healthcare staff tend to hold two contradictory ideas in relation to this group of patients. Firstly many of them acknowledge and understand their particular needs, and sometimes put great effort into crafting appropriate responses (and they also reported finding it hard to get those responses incorporated into ongoing operational procedures). At the same time, staff reported a reliance on the principle of equal treatment (as in the statement 'you treated them like any other Tom, Dick or Harry that came through the ward.' [28 p.549] The principle of equal treatment is a very important one, particularly in a public health system, but always carries a qualifier: 'in accordance with need'. In the case of Aboriginal patients, it seemed that the legitimacy of their particular needs (such as for interpreters, or for support in their often arduous journeys to receive care) was somehow compromised. We concluded that this collective ambivalence in the provision of healthcare rested on the broad social silence, discomfort and denial that characterises mainstream Australian thinking about the position and role of Aboriginal people in Australia, and our shared history of colonisation.

If denial and silence are part of the problem, skirting around it is unlikely to be part of the solution. But while change strategies need to be based on an analysis of how systemic racism really works (and who benefits), the most effective methods for change are not likely to rely primarily on earnest discussions of Whiteness [29] and privilege by clinical teams. In reflecting on the findings of the research cited above, and our experience of discussing them with team members, we came to the conclusion that the very anxiety that nonAboriginal staff feel in contemplating the question of discriminatory practice is a barrier that impedes action.

'Racism anxiety' is the term we use to describe this barrier. For those seeking to improve equity, it is a problem that staff tend to feel that their moral standing is under attack if the topic of discrimination is raised. 'I'm not racist' is the defensive position, and anxiety about this perceived allegation tends to deflect attention from the problems at hand. This is not hard to understand - racism is commonly seen as working at an individual rather than systemic level. The idea that policies and practices can be discriminatory without conscious intention by those implementing them or working within their rules is not widely understood. Indeed, the logical implication of the system's focus on cultural awareness training for staff is precisely that individuals are primarily responsible for discriminatory practice. And when staff perceive that they are being asked to first acknowledge a moral failing in themselves before they roll up their sleeves and fix the problem, it is not surprising that most people tend to put the issue in the too hard basket, or simply turn away. [30] Thus while it is essential that action to improve equity in healthcare for Aboriginal people is based on acknowledgement of the racism that is built in to the health system's policies, practices, protocols and programs, this is not enough. For effective action to improve access to equitable care, there needs to be a way of releasing staff from the paralysing grip of racism anxiety. We need to name it, acknowledge its power, and find ways to deal with it constructively. 


\section{Competence for Culturally Safe Care}

The evidence on improvement strategies indicates that existing approaches have been less effective than claimed, and systematic multi-level strategies are required. There are many concepts and approaches in this field. We suggest that two are essential. The first is cultural safety, which we define in this context as the patient's experience of care that is respectful of cultural identity and integrity. [31,32] The second is the competence of the healthcare organisation to deliver culturally safe care, which requires strategies, policies, practices and programs at all levels of the organisation that enable it, through its staff, to reliably provide care that is responsive to need and does no harm to patients' identity and cultural integrity. We prefer not to use the term cultural competence [33] in the current context, because it implies the need for healthcare organisations and their staff to become competent practitioners of a culture to which they don't belong.

Organisational competence for culturally safe care requires the effective implementation of practical measures to reduce discrimination, enhance respect for cultural identity, and remove barriers to access. Given the complexity of healthcare, and the wickedly specific requirements in each clinical area, we suggest that specific measures need to be developed, tested and shared by health services, within a supportive framework - a framework that encompasses all levels of the system that lie between high policy goals and the practice of healthcare staff. [34]

\section{Cultural awareness approaches}

Evaluation of the cultural awareness approach documents its lack of the desired impact. [35] These and other authors [36, p.1210] point out that the apparent failure of cultural awareness training seems predictable because it tends to both 'essentialise' Aboriginality and make 'other' Aboriginal people. The very act of giving health workers a sense of some knowledge of Aboriginal cultures keeps the focus on Aboriginality and away from the need for healthcare practice to be based on an understanding of the ways in which the mainstream system denigrates and discriminates against Aboriginal people. It also may encourage health workers to make assumptions about their Aboriginal patients as people who will conform with stereotypes, a practice that is not helpful to the quality of the healthcare relationship, or to diagnosis and treatment. Cultural awareness training may thus defeat its goal which is to enable the provision of care that treats Aboriginal patients as individuals, according to their needs, with respect and without prejudice.

\section{The evidence for 'cultural competence'}

The literature in relation to 'cultural competence' (CC) is growing, but the evidence of impact is not yet strong. Studies of the effectiveness of this approach for Indigenous people in Australia, New Zealand, Canada and the United States were found to be of questionable quality in a recent systematic review. [37] The main benefits reported were improved patient satisfaction and access to care, and improved confidence for health professionals. The main intervention strategies reported were training, culturally specific health services and increasing the Indigenous health workforce.

Bainbridge et al [34] found the formation of partnerships with local Aboriginal communities, as well as action to embed CC in governance, policies and programs, to be useful, and they suggested legislation or policy to entrench a requirement for attention to $\mathrm{CC}$, as is the case in the United States and New Zealand. In a recent systematic review of 19 reviews, Truong, Paradies and Priest [38] examined the evidence for cultural competency, which they defined to include interventions (principally training) aimed at healthcare staff, as well as those applied at the level of the organisation or system. They found some evidence of a link between the cultural competence of organisations and that of their staff (but this is a long way short of evidence of safer care). They found moderate evidence of improvement in provider knowledge/skill and healthcare access/ usage, but weaker evidence for improvements in patient or client outcomes. They also found that few of the reported interventions included attention to racism and discrimination, and only some included attention to selfreflection and awareness of one's professional and social culture. [39]

While there is, as yet, little evidence of outcomes from organisational cultural competency approaches in the mainstream Australian health system, recent research supports two important foundational ideas: the first is to base approaches on an explicit recognition of the ongoing impact of racism and colonisation; and the second is to use a comprehensive and sustained set of strategies in policy, practice, programs, training and reward systems for staff. [40-46]

\section{Evidence of mainstream responses}

There is reason to believe that since the transfer of responsibility for Aboriginal health from the Aboriginal and Torres Strait Islander Commission to the health portfolio in 1995, there has been slow and patchy but sustained growth in efforts within the mainstream health system to improve 
access and quality of care for Aboriginal people. This view is supported by Australian research evidence that experiences of racism are less common in healthcare than in other settings. [26,47] Examples include the national Better Cardiac Care measures, [48] the sustained effort by the Hunter New England Health Service [49], and increasing attention by professional groups and organisations to the implications for practice in cardiovascular health. [50]

\section{What is to be done?}

As always in healthcare, knowledge of problems and the development of strategies to address them will emerge from practice; and a supportive environment is needed to enable solutions to be embedded rather than lost. This is the fundamental purpose of quality improvement methods, and they are being used successfully to improve access and quality of care for Aboriginal patients. For example, Kelly and colleagues [51] report on changes in end-of-life care for renal patients, based on the careful work of a group of renal nurses to map patient journeys, followed by the development of new pathways and the resources to support them. We suggest that in the case of Aboriginal patient care, this practice-based knowledge often lacks the necessary supportive organisational environment; and creating or strengthening it is a challenge that healthcare leaders can meet.

There are several frameworks that can guide health services, including at least two developed in Australia. [52,53] These frameworks, and the evidence cited above, reinforce the importance of two foundational principles for health services.

\section{Work in alliance with local ACCHOs and community organisations}

It is essential for health services to work actively and collaboratively with the Aboriginal and Torres Strait Islander communities they serve. Local health and community organisations provide an existing structure and networks to enable this engagement.

Aboriginal Community Controlled Health Organisations (ACCHOs) and some other Indigenous-specific teams and organisations play a critical role in providing culturally and clinically safe primary healthcare to their patients and communities, and bring essential expertise. They address the negative impact of continuing discrimination, and work with mainstream health services and other health institutions to support efforts to improve mainstream care. [54-56]

\section{Action needs to be targeted, but the opportunities are at} all levels

The second foundational principle is the need for coordinated attention across the organisation. The challenge is to understand and then act to change the often invisible ways in which Aboriginal people are excluded and discriminated against. The direct caring relationship between staff and patients is where culturally safe care is delivered, but the barriers to competence for culturally safe care are in policies, practices, protocols and programs (or their absence) throughout the organisation. This doesn't mean organisations have to try to change everything at once, but rather that they need to analyse their own problems, and prioritise action to remove or reduce them.

And most importantly, strategies need to be tested and the knowledge about what works needs to be shared. Solutions and strategies will always need to be locally planned and implemented, but they will be more effective if informed by evidence and the experience of others. As always, more research is needed, and in this case, comparative intervention studies of known methods and approaches are a high priority.

\section{Competing interests}

The authors declare that they have no competing interests.

\section{References}

1. Condon JR, Barnes T, Armstrong BK, Seval-Nayagam S, Elwood JM. Stage at diagnosis and cancer survival for Indigenous Australians in the Northern Territory. Med J Aust. 2005;182(6):277-80.

2. Davidson PM, Jiwa M, Digiacomo ML, McGrath SJ, Newton PJ, Durey AJ, Bessarab DC, Thompson SC. The experience of lung cancer in Aboriginal and Torres Strait Islander peoples and what it means for policy, service planning and delivery. Aust Health Rev. 2013; 37(1):70-8.

3. Boffa JD. Cancer care for Indigenous Australians. Med J Aust. 2008; 188(10):560-561.

4. Better hospital care for Aboriginal and Torres Strait Islander people experiencing heart attack. National Heart Foundation of Australia and Australian Healthcare and Hospitals Association; 2010.

5. Australian Institute of Health and Welfare. Spatial variation in Aboriginal and Torres Strait Islander people's access to primary health care. 2015. AlHW cat. no. IHW 155.

6. Artuso S, Cargo M, Brown A, Daniel M. Factors influencing health care utilisation among Aboriginal cardiac patients in central Australia: a qualitative study. BMC Health Serv Res. 2013;6(13):83.

7. Cass A, Devitt J, Preece C, Cunningham J, Anderson K, Snelling P, Eris J, Ayanian J. Barriers to access by Indigenous Australians to kidney transplantation: the IMPAKT study. Nephrol. 2004;9(s4): S144-6.

8. Preston-Thomas A, Cass A, O'Rourke P. Trends in the incidence of treated end-stage kidney disease among Indigenous Australians and access to treatment. Aust N Z J Public Health. 2007;31(5): 419-21. 
9. Devitt J, Cass A, Cunningham J, Preece C, Anderson K, Snelling P. Study Protocol - Improving Access to Kidney Transplants (IMPAKT): A detailed account of a qualitative study investigating barriers to transplant for Australian Indigenous people with end-stage kidney disease. BMC Health Serv Res [Internet]. 2008 [cited 2015 Aug 19]; 8(31). Available from: http://www.biomedcentral.com/ $1472-6963 / 8 / 31$

10. Anderson K, Devitt J, Cunningham J, Preece C, Jardine M, Cass A. If you can't comply with dialysis, how do you expect me to trust you with transplantation? Australian nephrologists' views on indigenous Australians'”non-compliance' and their suitability for kidney transplantation. Int J Equity Health. 2012;11(1). DOI: 10.1186/ 1475-9276-11-21

11. Australian Institute of Health and Welfare. Australian hospital statistics 2010/11. 2012. AlHW cat. no. HSE117.

12. Australian Institute of Health and Welfare. Australian hospital statistics 2012-13. 2014. AlHW cat. no. HSE 145.

13. Australian Institute of Health and Welfare. Aboriginal and Torres Strait Islander health performance framework, 2008 Report: Detailed analyses. 2008. AlHW cat. No. IHW 22.

14. Wright L. They just don't like to wait!': A comparative study of Aboriginal and non-Aboriginal people who do not wait for treatment or discharge themselves against medical advice from rural emergency departments. Port Macquare: New South Wales Health; 2007.

15. Einsiedel LJ, van lersel E, Macnamara R, Spelman T, Heffernan M, Bray L, Morris H, Porter B, David A. Self-discharge by adult Aboriginal patients at Alice Springs Hospital, Central Australia: insights from a prospective cohort study. Aust Health Rev. 2013;37(2): 239- 245.

16. Department of Health and Ageing. The state of our hospitals June 2009 report. Canberra: Australian Government; 2009.

17. Australian Institute of Health and Welfare. The health and welfare of Australia's Aboriginal and Torres Strait Islander people: an overview. Canberra: Australian Government; 2011.

18. Lawrence M, Dodd Z, Mohor S, Dunn S, de Crespigny C, Power C Mackean L. Improving the patient journey. Darwin: Cooperative Research Centre for Aboriginal Health; 2009.

19. Strengthening cardiac rehabilitation and secondary prevention for Aboriginal and Torres Strait Islander peoples [Internet]. Canberra: National Health and Medical Research Council; 2005. Available from: www.nhmrc.gov.au/publications/synopses/_files/ind1.pdf

20. Purdie N, Dudgeon P, Walker R. Working together: Aboriginal and Torres Strait Islander mental health and wellbeing principles and practice. Canberra: Department of Health and Ageing; 2010.

21. Eckermann A-K, Dowd T, Chong E, Nixon L, Gray R, Johnson S. Binan Gonnj: bridging cultures in Aboriginal health. Elsevier Churchill Livingstone, Marrickville, NSW; 2006.

22. Alford K. Comparing Australian with Canadian and New Zealand primary care health systems in relation to indigenous populations: literature review and analysis. Melbourne: Onemda VicHealth Koori Health Unit, The University of Melbourne; 2005.

23. Rogers GD, Barton C, Pekarsky B, Lawless A, Oddy J, Hepworth R, Beilby J. Caring for a marginalised community: the costs of engaging with culture and complexity. Med J Aust. 2005;183:S59-63.

24. Taylor K, Guerin P. Health care and Indigenous Australians: cultural safety in practice. Melbourne: Palgrave Macmillan; 2010.

25. Cass A, Lowell A, Christie M, Snelling P, Flack M, Marrngnanjin B, Brown I. Sharing true stories: improving communication between Aboriginal patients and healthcare workers. Med J Aust. 2002; 176:466- 70 .

26. Kelaher M, Ferdinan, $A$, Paradies Y. Experiencing racism in health care: the mental health impacts for Victorian Aboriginal Communities. Med J Aust. 2014;201(1):44-47.
27. Vos T, Barker B, Begg S, Stanley S, Lopez AD. Burden of disease and injury in Aboriginal and Torres Strait Islander Peoples: the Indigenous health gap. Int J Epidemiol. 2009;38:470-477.

28. Dwyer J, Willis E, Kelly J. Hospitals caring for rural Aboriginal patients: holding response and denial. Aust Health Rev. 2014;38(5): 546-51.

29. Kowal E, Paradies Y. Race and Culture in Health Research: A Facilitated Discussion. Darwin: Cooperative Centre for Aboriginal and Tropical Health; 2003.

30. Wilson AM, Magarey AM, Jones M, O'Donnell K, Kelly J. Attitudes and characteristics of health professionals working in Aboriginal health [Internet]. Rural and Remote Health. 2015 [cited 2015 Aug 31]; 15:2739. Available from: http://www.rrh.org.au/publishedarticles/ article_print_2739.pdf

31. Papps E, Ramsden I. Cultural Safety in Nursing: the New Zealand Experience. Int J Qual Saf Health Care. 1996; 8(5):491-497.

32. Downing R, Kowal E. A postcolonial analysis of indigenous cultural awareness training for health workers. Health Sociol Rev. 2011; 20(1):5-15.

33. Cross T, Bazron B, Dennis K, Isaacs M. Towards A Culturally Competent System of Care. Washington: Georgetown University Child Development Center, CASSP Technical Assistance Center; 1989.

34. Bainbridge R, McCalman J, Clifford A, Tsey K. Cultural competency in the delivery of health services for Indigenous people. Closing the Gap Clearinghouse. Issues paper no. 13. Canberra: AIHW and AIFS; 2015.

35. Downing R, Kowal E, Paradies Y. Indigenous cultural training for health workers in Australia. Int J Qual Health Care. 2011; 23(3):247-257.

36. Williams DR, Mohammed SA. Racism and Health II: A Needed Research Agenda for Effective Interventions. Am Behav Sci. 2013; 57(8:SI):1200-1226.

37. Clifford A, McCalman J, Bainbridge R, Tsey K. Interventions to improve cultural competency in health care for Indigenous peoples of Australia, New Zealand, Canada and the USA: a systematic review. Int J Qual Healthcare. 2015;27(2):89-98.

38. Truong $M$, Paradies $Y$, Priest N. Interventions to improve cultural competency in healthcare: a systematic review of reviews [Internet]. BMC Health Serv Res. 2014;14:99. Available from: http://www. biomedcentral.com/1472-6963/14/99

39. Kowal E, Franklin H, Paradies Y. Reflexive antiracism: a novel approach to diversity training. Ethnicities. 2013;13(3):316-337.

40. Durey A, Thompson SC. Reducing the health disparities of Indigenous Australians: time to change focus [Internet]. BMC Health Serv Res. 2012;12(151).

41. Durey A, Thompson SC, Wood M. Time to bring down the twin towers in poor Aboriginal hospital care: addressing institutional racism and misunderstandings in communication. Intern Med J . 2012;42(1):17-22.

42. Rix EF, Barclay L, Stirling J, Tong A, Wilson S. The perspectives of Aboriginal patients and their health care providers on improving the quality of hemodialysis services: a qualitative study. Hemodial Int. 2015;19(1):80-89.

43. Rix EF, Barclay L, Stirling J, Tong A, Wilson S. Beats the alternative but it messes up your life': Aboriginal people's experience of haemodialysis in rural Australia [Internet]. BMJ Open. 2014;4(9). Available from: http://bmjopen.bmj.com/content/4/9/e005945.full

44. Rix EF, Barclay L, Wilson S, Stirling J, Tong A. Service providers' perspectives, attitudes and beliefs on health services delivery for Aboriginal people receiving haemodialysis in rural Australia: a qualitative study. BMJ Open. 2013;3(10). 
45. Lau P, Pyett P, Burchill M, Furler J, Tynan M, Kelaher M, Liaw S-T. Factors influencing access to urban general practices and primary health care by Aboriginal Australians - A qualitative study. AlterNative. 2012;8(1):66-84.

46. Reibel T, Walker R. Antenatal services for Aboriginal women: the relevance of cultural competence. Qual Prim Care. 2010;18:65-74.

47. Willis E, Dwyer J, Owada K, Couzner L, Wainer J. Urban Aboriginal women's expectations of clinical care during treatment for a gynaecological cancer: exploring the gaps in the research. Aust Health Rev. 2011:35(1):1-5.

48. Australian Institute of Health and Welfare. Better Cardiac Care measures for Aboriginal and Torres Strait Islander people: First National Report. [cited 18 August 2015]. Canberra: AlHW; 2015. Available from: <http://www.aihw.gov.au/publication-detail/ ?id=60129551940>

49. Closing the gap in a regional health service in NSW: a multi-strategic approach to addressing individual and institutional racism. Hunter New England Health Aboriginal and Torres Strait, Islander Strategic Leadership Committee; New South Wales Public Health Bulletin. 2012;23(3-4):63-67.

50. Davidson PM, Maclsaac A, Cameron J, Jeremy R, Mahar L, Anderson I. Problems, solutions and actions: addressing barriers in acute hospital care for indigenous Australians and New Zealanders. Heart Lung Circ. 2012;21(10):639-43.

51. Kelly J, Wilden C, Herman K, Martin G, Russell C, Brown S. Bottling knowledge and sharing it - using patient journey mapping to build evidence and improve Aboriginal renal patient care, Renal Society of Australasia Journal. 2016:12(10):48-55.

52. Tynan M, Atkinson $P$, Smullen F, Stephens K. Developing an Aboriginal Cultural Competence Framework and Audit Tool for health services in regional Victoria: lessons for implementation. Aust NZJ Public Health. 2013;37(4):392-3.

53. Marrie A, Marrie H. A Matrix for Identifying, measuring and monitoring Institutional racism within Public Hospitals and Health Services [Internet]. Gordonvale: Burkal Consultancy Services; 2014. Available from: http://www.avidstudy.com/wpcontent/uploads/ 2015/08/Matrix-Revised-2-9-14.pdf
54. Panaretto K, Wenitong M, Button S, Ring I. Aboriginal community controlled health services: leading the way in primary care. Med J Aust. 2014;200(11):649-652.

55. Baba JT, Brolan CE, Hill PS. Aboriginal medical services cure more than illness: a qualitative study of how Indigenous services address the health impacts of discrimination in Brisbane communities. Int J Equity Health. 2014;10(13):56.

56. Freeman T, Edwards T, Baum F, Lawless A, Jolley G, Javanparast $\mathrm{S}$, Francis T. Cultural respect strategies in Australian Aboriginal primary health care services: beyond education and training of practitioners. Aust NZJ Public Health. 2014;38(4):355-61. 